\title{
Future foods for risk-resilient diets
}

\author{
Asaf Tzachor ${ }^{1,2} \bowtie$, Catherine E. Richards ${ }^{1,3}$ and Lauren Holt ${ }^{1}$
}

Future foods, such as microalgae, mycoprotein and mealworm, have been suggested as nutritious and sustainable dietary options. Here we consider one of the most profound, yet neglected, benefits of future foods farming systems-their potential to provide essential nutrition in the face of systemic disturbances-and discuss major barriers to realizing this prospect.

$\Lambda$ series of shocks, including the worst pandemic for a century, has recently highlighted the fragility of agri-food systems. In an attempt to contain the spread of COVID-19, authorities imposed unprecedented movement restrictions that disrupted agri-food production and supply chains. As societies coped with the pandemic, additional stressors-such as swarms of desert locust (Schistocerca gregaria) in East Africa, outbreaks of African swine fever in Asia and Europe, tropical cyclones in the Indian subcontinent ${ }^{1}$ and wildfires, droughts and a derecho in North America $^{2}$ - exacerbated food insecurity in regions experiencing severe undernutrition and undermined just-in-time supply chains in relatively food-secure regions.

The concurrent crises accentuated the risk of hinging our diets on plant-source foods (PSF) and animal-source foods (ASF) produced through techniques that are innately exposed to various acute and chronic stresses. Conventional farming systems of PSFincluding wheat, maize, rice and soybean-are naturally susceptible to multiple and unmitigated abiotic pressures, such as alternations in edaphic and atmospheric factors ${ }^{3,4}$. Biotic stressors, including ectoparasites, endoparasites and pathogens, further threaten crop yields ${ }^{5}$. Conventional farming systems of terrestrial ASF-namely dairy cattle, beef cattle, pigs, broiler chicken and laying hens-are also vulnerable to the risks and uncertainties of PSF farming, as most livestock rely on terrestrial vascular plants for feed. Typically farmed under concentrated animal feeding operations, livestock are at risk of skeletal weakness, deformities and premature culling $^{6}$, whereas high stocking densities elevate exposure to increased pathogen mutation rates and other biotic risks ${ }^{7}$.

Anthropogenic climate change is projected to exacerbate a number of these threats ${ }^{8}$, with graver consequences in developing regions ${ }^{9}$. Likewise, fluctuations in the biophysical parameters of conventional farming may hinder production performance. Although institutional interventions in such instability may help, abrupt variations in food production often lead to inconsistency in the timely and equitable provision of essential nutrients, with potentially long-lasting effects among vulnerable populations ${ }^{10}$.

Here, we show that future foods farming systems constitute a pathway for mitigating global malnutrition. As edible items, future foods are increasingly recognized as nutritious and more sustainable alternatives to PSF and ASF ${ }^{11}$. We argue that future foods should be appraised from a different vantage point, one that goes beyond their ecological benefits and has not been discussed thus far: their potential to deliver risk-resilient diets. In line with previous studies $^{12}$, we define a risk-resilient diet as one consistent in the provision of essential macro- and micronutrients in the face of disturbances, whether they be global or local, predictable or sudden.

\section{Promising future foods farming systems}

Food technology opens up many possibilities for alternative and risk-reduced farming. Recent studies indicate the viability of cultivating several future foods at scale in controlled environments ${ }^{11}$.

Microalgae including chlorella (Chlorella vulgaris) and spirulina (Arthrospira platensis) are a promising food source by dint of their fast growth rates as unicellular organisms. Recent studies report novel configurations in which microalgae are cultivated in a liquid medium in closed photobioreactors and irradiated by light-emitting diodes to achieve a high photosynthetic photon flux at optimized wavelengths, thereby improving the efficiency of photosynthesis ${ }^{13}$.

For macroalgae, including sugar kelp (Saccharina latissima), and mussels (Mytilus spp.), advances in aquaculture have seen the development of industrial-scale farms in coastal and offshore locations, outside native growth zones. New integrated multitrophic aquaculture systems promise a consistent feedstock, in situ, with the co-benefit of recycling aquaculture waste ${ }^{14}$.

Mycoprotein derived from filamentous fungi, including Fusarium venenatum, has been produced for use in commercially available alternative meat products for several decades. Fungal biomass is cultivated using continuous-flow aerobic fermentation in temperature- and $\mathrm{pH}$-controlled reactors with carbohydrate and nutrient substrate growth media ${ }^{15}$.

Insect larvae, including black soldier fly (Hermetia illucens), house fly (Musca domestica) and mealworm beetle (Tenebrio molitor), may also be effectively produced at scale. Although nuanced for different species, typical larval breeding systems consist of similar processes, assisted by automated equipment contained in stackable multicompartment units. These systems leverage natural migratory habits between growth stages and can also provide ancillary benefits of recycling organic waste as feedstock ${ }^{16}$.

On other frontiers, food technology has not yet matured to support scalable production. Cultured meat, also referred to as cellular agriculture, is one example. Although cultured meat prototypes have been successfully developed and pilot plants are under construction, the technology is still relatively nascent, energy intensive and not yet economically viable ${ }^{17}$.

The closed-environment conditions offered by many of these future foods farming systems enable a higher degree of control over culture processes and mitigate many of aforementioned perils of conventional PSF and ASF farming (Fig. 1).

\section{Resilience benefits}

In considering the potential of future foods to deliver resilient diets, we assert three important resilience advantages associated with future foods farming systems: reducing exposure to biotic and 


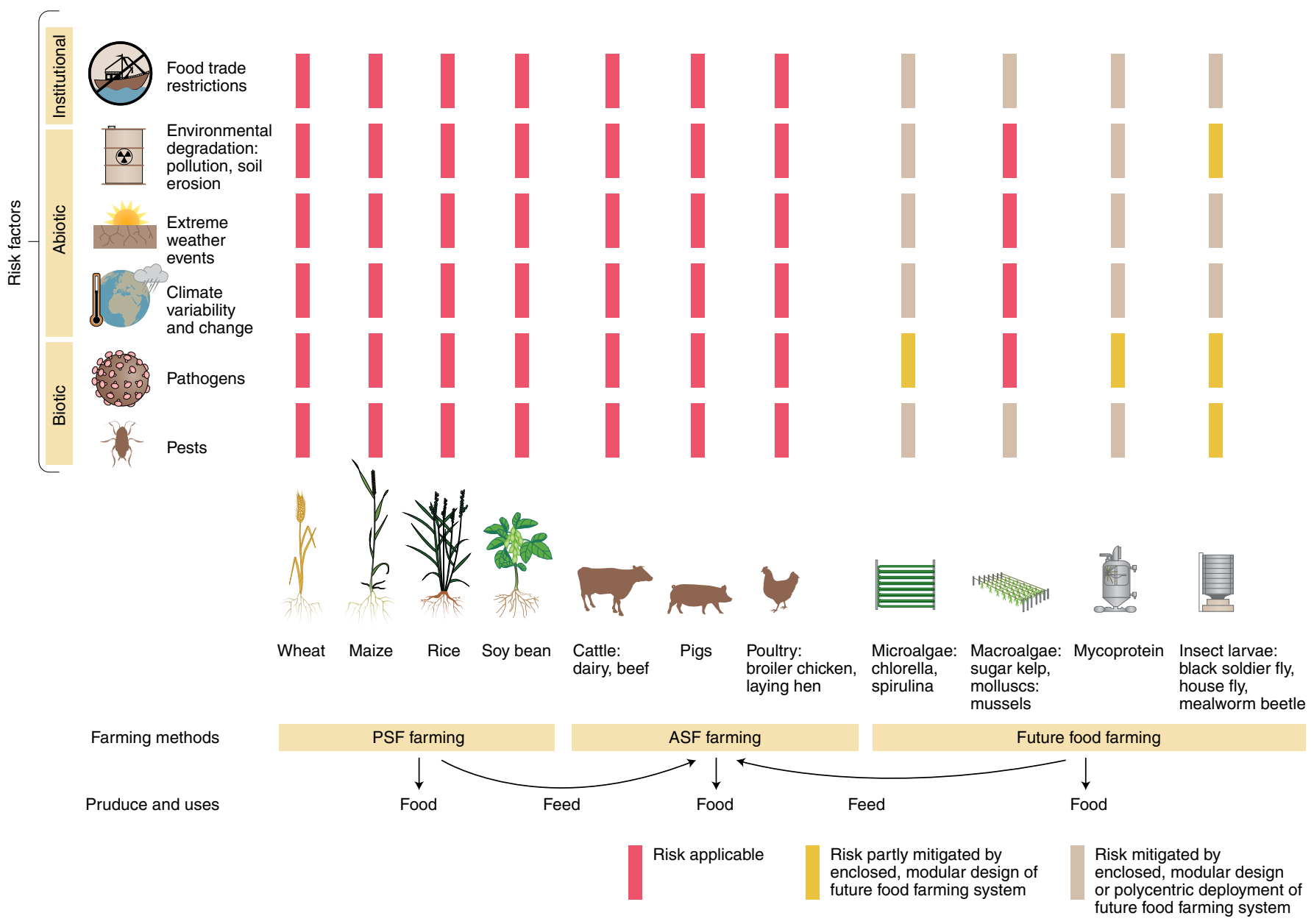

Fig. 1 | Current risk landscape of ASF, PSF and future foods farming systems. All farming systems are assessed in their current state of development, as described in this Perspective. Each bar denotes a hazard threatening a particular farming system. The enclosed, modular design or polycentric deployment of future foods farming systems can mitigate the risks to which the open-environment farming systems of traditional ASF and PSF are vulnerable.

abiotic risk factors; fostering modularity to contain farming process failures, decouple risks and adjust yields; and providing dietary consistency of essential nutrition through globally decentralized and locally distributed food networks.

Closed environment. At present, several future foods can be cultivated in closed systems, including stackable, multicompartment nurseries or heterotrophic and autotrophic bioreactors. Largely unconstrained by environmental conditions that otherwise jeopardize PSF and ASF yields, future foods farming systems can maintain steady internal physical, chemical and biological conditions, and therefore consistent and efficient production performance ${ }^{11}$.

Moreover, by separating essential nutrition production from ASF supply chains-namely livestock meat processing in slaughterhouses-future foods farming systems prevent the risk of bacterial and faecal matter contamination, such as Salmonella and Escherichia coli $^{18}$. Similarly, by replacing ASF with future foods, the risk of botulism caused by bacterium Clostridium botulinum in animal carcasses is eliminated.

Modular design. State-of-the-art future foods farming systems are typically divided into discrete, standardized, identical production units referred to as modules. A modular architecture, particularly a modular open systems approach, offers manifold functional benefits $^{19}$. In the context of risk resiliency, modularity allows for flexible responses to unexpected disruptions. Production components that cultivate future foods in parallel, and decoupled from each other, can be easily disassembled and replaced without hampering overall production consistency ${ }^{20}$

This feature is particularly important, as potential contaminations are confined to the in vitro environment of each production unit and prevented from spilling over to other cultivation components. It also provides operational redundancy in so far as edible biomass may proceed from multiple smaller operating units instead of a few larger ones; this decreases the dependence on each unit should it malfunction, while increasing system-wide stability ${ }^{21}$. For instance, the biomass production of the fly $H$. illucens exploits these advantages by rearing in small, stand-alone greenhouses ${ }^{16}$.

Another benefit of a modular architecture is the ability to adjust production to meet oscillating demand. Production units can be supplemented or extracted, and processes can be decelerated, accelerated, halted or renewed, as required. This is particularly useful if supply and processing chains are immobilized, or if demand plummets or peaks ${ }^{22}$. For example, cellular metabolism rates may be regulated in photobioreactors to adjust microalgal biomass yield ${ }^{13}$.

Polycentric food networks. Future foods farming systems provide the opportunity to rapidly decentralize and localize the production and supply of essential nutrients. In contrast to conventional farming, concentrated in regions of favourable environmental 
conditions, future foods farming systems can be deployed in various geographies.

Global decentralization and local distribution should enhance dietary resilience in four ways, which coincide neatly with other benefits mentioned here and in the resilience literature ${ }^{23}$. First, future foods farming systems foster geographical diversification of farming, reducing the consequences of spatial risks and compartmentalizing potential crises. With these systems deployed extensively, the effects of a derecho in North America, for instance, will not cascade to undermine nutritional security afield.

Second, distribution may mitigate the risk of malnutrition among isolated communities in remote regions with limited access to PSF and ASF, such as in Pacific Island states where feeble agriculture and consumption of nutrient-poor foods contribute to stunting in children, and iron-deficiency anaemia in women of reproductive age ${ }^{24}$.

Third, future foods farming systems, such as mealworm breeding greenhouses and spirulina photobioreactors, require shorter supply lines and can be placed in, or near to, urban centres ${ }^{25}$. This avoids the risk of international blockades and export sanctions, such as those experienced during the coronavirus pandemic ${ }^{26}$. Fourth, and consequently, future foods farming systems may respond faster to fluctuations in prices and demand, moderate the bullwhip effect and be less prone to variability and volatility.

Concomitant with these benefits, globally decentralized and locally distributed farming can prompt the democratization of food systems, empowering consumers to become prosumers. This may encourage citizen-centred innovations in designing and refurbishing cultivation apparatus, as well as in the development of future foods products ${ }^{27}$, using them as whole foods and ingredients and additives, including as antioxidant and anti-inflammatory agents.

\section{Deployment challenges}

Technologies that underpin future foods farming systems have taken considerable strides forwards in the recent past. These advances are promising for the provision of risk-resilient diets, as nutritional diversification from PSF and ASF to future foods can be achieved. However, while future foods farming systems offer a great deal, we must be careful not to overestimate their potential. There are a number of barriers still to be addressed if these benefits are to be realized at scale.

Technical barriers. Bioreactors for microalgae, multicompartment nurseries for insects and aerobic fermentation reactors for fungi are effective in producing edible biomass, but depend on energy for light and heating. As future foods farming systems are deployed more widely, ensuring a reliable and consistent energy supply becomes more challenging. In many regions, specifically those at crisis-level hunger, sufficient energy production capacity cannot yet be supplied.

Even if sufficient and steady electricity is available, future foods farming systems may rely on non-renewable and polluting energy sources, which ultimately undermines their sustainability premise. Therefore, integration with renewable energy sources should be prioritized.

The integration of other technologies should likewise be pursued, including emerging applications for production capacity, such as sensor technologies, and food safety monitoring, such as nanomaterial-based assays for analysis of contaminants.

Future foods farming systems face some of the same limitations as conventional farming. Suitable space for production units and associated infrastructure, such as biorefineries, is required to scale up production capacity. Moreover, these systems involve some kind of traditional agricultural input, such as concentrated compounds of nitrogen, phosphorus and trace elements, to ensure consistent yields. For instance, fermentation of fungus $F$. venenatum requires carbohydrates, such as fructose culture substrates, thereby remaining dependent on the PSF supply chain. In food insecure regions, affordable access to these inputs can prove difficult ${ }^{28}$.

Although the enclosed, modular architecture of future foods farming systems may mitigate many risks, biotic risks may persist to some degree. For instance, bacteria and fungi toxins may result in stock crashes in microalgae and mycoprotein production. Insect rearing may be degraded by parasites or bioaccumulation of chemicals and heavy metals resulting from the use of organic waste as feedstock.

Furthermore, as relatively new technologies, risk analysis of future foods farming systems remains incomplete. There may be new contaminants associated with their unique environments that are yet to be identified. Farming system integrity protocols and monitoring techniques are essential to maintain safe and stable production $^{29}$.

Some future foods farming systems, such as submerged aquaculture farms, remain located in open environments at present. These systems are vulnerable to alterations in environmental conditions, including exposure to pathogens, such as seawater bacteria Vibrio parahaemolyticus, plastic pollution, toxin bioaccumulation and variation in sea temperatures ${ }^{30}$. Further research and technological development of enclosed versions of these systems, including onshore and urban aquaculture farms, could mitigate these hazards.

Institutional barriers. Future foods farming systems require new technical expertise and considerable financial investment. This makes them less accessible to lower-income countries, where public sector subsidization or official development assistance may be necessary, compared with higher-income countries, where entrepreneurial investment is concentrated ${ }^{13}$.

Similarly, regulatory approval of new food products often demands substantial resources, including toxicology studies, and standards may differ between countries. Regulatory approval may also prove to be an obstacle in realizing ancillary benefits of recycling excretory substances, such as for insect feedstock.

Finally, while we specifically consider farming systems, the provision of future food products requires quality control along all steps of the supply chain, including downstream processing and biorefining. Consideration must also be given to gastronomic preferences, including the appeal and acceptance of future foods such as insect larvae, which are atypical in some cultures.

\section{Conclusion}

Future foods systems have the potential to provide a healthy and more environmentally sustainable diet-that is, one that provides essential and balanced nutrition with reduced environmental impacts. Such systems could provide resilient diets, consistent in the supply of essential nutrition in the face of acute biotic and abiotic stressors, as well as institutional disruptions.

In light of the coronavirus pandemic-an exemplar systemic risk event-and our increasing knowledge of structural risks, uncertainties and cascading failures in the global agri-food system, we maintain that a risk-resilient diet is essential to ensure short- and long-term food security. Attaining enhanced and robust food security into the future will require a sensitive balance of developments within the traditional, globalized agri-food system alongside integration of emerging food technologies that leverage enclosed, modular configurations and localized, polycentric deployment.

We urge scientists, engineers, investors and policymakers to consider future foods as a malnutrition mitigation pathway. This includes identifying and openly discussing underpinning challenges, as well as exploring how future foods farming systems can be rapidly and cost-effectively deployed in response to current instabilities and in anticipation of future hazards. 


\section{Data availability}

The data that support the findings of this study are available from the corresponding author upon request.

Received: 15 August 2020; Accepted: 31 March 2021; Published online: 13 May 2021

\section{References}

1. FAO Warns Multiple Impact of Viruses, Plagues and Economic Damage Will Fuel Hunger in Asia and the Pacific (Reliefweb, United Nations Office for the Coordination of Humanitarian Affairs, 2020).

2. Summer 2020 Ranked as One of the Hottest on Record for US (National Oceanic and Atmospheric Administration, 2020).

3. Minhas, P. S., Rane, J. \& Pasala, R. K. (eds) Abiotic Stress Management for Resilient Agriculture (Springer, 2017).

4. Borrelli, P. et al. An assessment of the global impact of 21st century land use change on soil erosion. Nat. Commun. 8, 2013 (2017).

5. Singla, J. \& Krattinger, S. G. in Encyclopedia of Food Grains 2nd edn (eds Wrigley, C. W. et al.) 388-392 (Academic, 2016).

6. Meseret, S. A review of poultry welfare in conventional production system. Livest. Res. Rural Dev. 28, 234 (2016).

7. Alvarado, C. S. et al. Seasonal changes in airborne fungi and bacteria at a dairy cattle concentrated animal feeding operation in the southwest United States. J. Environ. Health 71, 40-45 (2009).

8. Campbell, B. M. et al. Reducing risks to food security from climate change. Glob. Food Secur. 11, 34-43 (2016)

9. Lancelot, R., De La Rocque, S. \& Chevalier, V. Bluetongue and Rift Valley fever in livestock: a climate change perspective with a special reference to Europe, the Middle-East and Africa. In Proceedings of the International Conference on Livestock and Global Climate Change (Cambridge Univ. Press, 2008).

10. Evans, P., Bennett, D. \& Jennings, R. C. (eds). Feeding 9 Billion-The Contribution of New Genetic Technologies to Global Food Production (Banson, 2014).

11. Parodi, A. et al. The potential of future foods for sustainable and healthy diets. Nat. Sustain. 1, 782-789 (2018).

12. Tendall, D. M. et al. Food system resilience: defining the concept. Glob. Food Secur. 6, 17-23 (2015).

13. Nwoba, E. G., Parlevliet, D. A., Laird, D. W., Alameh, K. \& Moheimani, N. R. Light management technologies for increasing algal photobioreactor efficiency. Algal Res. 39, 101433 (2019).

14. Buck, B. H. et al. State of the art and challenges for offshore integrated multi-trophic aquaculture (IMTA). Front. Mar. Sci. 5, 165 (2018).

15. Hashempour-Baltork, F., Khosravi-Darani, K., Hosseini, H., Farshi, P. \& Reihani, S. F. S. Mycoproteins as safe meat substitutes. J. Cleaner Prod. 253, 119958 (2020)

16. Makker, H. P. S., Tran, G., Heuze, V. \& Ankers, P. State-of-the-art on use of insects as animal feed. Anim. Feed Sci. Technol. 197, 1-33 (2014).

17. Post, M. J. et al. Scientific, sustainability and regulatory challenges of cultured meat. Nat. Food 1, 403-415 (2020).

18. Tutenel, A. V., Pierard, D., Van Hoof, J. \& De Zutter, L. Molecular characterization of Escherichia coli $\mathrm{O} 157$ contamination routes in a cattle slaughterhouse. J. Food Prot. 66, 1564-1569 (2003).
19. Gershenson, J. K., Prasad, G. J. \& Zhang, Y. Product modularity: definitions and benefits. J. Eng. Des. 14, 295-313 (2003).

20. Gu, P. Product Architecture. In CIRP Encyclopedia of Production Engineering (eds Laperrière, L. \& Reinhart, G.) (Springer, 2014).

21. Dahlgren, E., Göçmen, C., Lackner, K. \& Van Ryzin, G. Small modular infrastructure. Eng. Econ. 58, 231-264 (2013).

22. Ulrich, K. in Management of Design (eds Dasu S. \& Eastman C.) 219-231 (Springer, 1994)

23. Biggs, R., Schlüter, M. \& Schoon, M. L. (eds) Principles for Building Resilience-Sustaining Ecosystem Services in Social-Ecological Systems (Cambridge Univ. Press, 2015)

24. Albert, J. et al. Malnutrition in rural Solomon Islands: an analysis of the problem and its drivers. Matern. Child Nutr. 16, e12921 (2020).

25. Specht, K. et al. How will we eat and produce in the cities of the future? From edible insects to vertical farming - a study on the perception and acceptability of new approaches. Sustainability 11, 4315 (2019).

26. Bakalis, S. et al. How COVID-19 changed our food systems and food security paradigms. Curr. Res. Food Sci. 3, 166-172 (2020).

27. Democratizing food systems. Nat. Food 1, 383 (2020)

28. Kelly, V., Adesina, A. A. \& Gordon, A. Expanding access to agricultural inputs in Africa: a review of recent market development experience. Food Policy 28, 379-404 (2003).

29. Gałęcki, R. \& Sokół, R. A parasitological evaluation of edible insects and their role in the transmission of parasitic diseases to humans and animals. PLoS ONE 14, e0219303 (2019).

30. Turner, L. et al. Pathogenic marine microbes influence the effects of climate change on a commercially important tropical bivalve. Sci. Rep. 6 , 32413 (2016).

\section{Acknowledgements}

C.E.R. was supported by a grant from Templeton World Charity Foundation, Inc. The opinions expressed in this publication are those of the author(s) and do not necessarily reflect the views of Templeton World Charity Foundation, Inc. We thank K. Atanasova for the graphic design of Fig. 1.

\section{Author contributions}

All authors contributed to the inception and development of this paper. A.T. and C.E.R. handled the revision process.

\section{Competing interests}

The authors declare no competing interests.

\section{Additional information}

Correspondence should be addressed to A.T.

Peer review information Nature Food thanks David Willer and the other, anonymous, reviewer(s) for their contribution to the peer review of this work.

Reprints and permissions information is available at www.nature.com/reprints. Publisher's note Springer Nature remains neutral with regard to jurisdictional claims in published maps and institutional affiliations.

(C) Springer Nature Limited 2021 